\title{
THIONE-THIOL TAUTOMERISM OF THIOUREA LIGANDS ON SILICA SURFACE
}

\author{
Chuiko Institute of Surface Chemistry of National Academy of Sciences of Ukraine \\ 17 General Naumov Str., Kyiv, 03164, Ukraine, E-mail: osmirnova@isc.gov.ua
}

\begin{abstract}
Quantum chemical calculations of the IR spectra of the thione and thiol forms of $N, N^{\prime}$-dimethylthiourea and silica surface fragment with composition $(\mathrm{HO})_{3} \mathrm{SiCH}_{2} \mathrm{NHC}(\mathrm{S}) \mathrm{NHCH}_{3}$ (density functional theory method, B3LYP/6-31G $(d, p))$ make it possible to fix indicator absorption bands of the tautomers. The presence of absorption bands at $1586 \mathrm{~cm}^{-1}$ (or $1607 \mathrm{~cm}^{-1}$ in the case of surface fragment) reveals the thione form whereas appearance of the intensive absorption bands at 1714 (1707) $\mathrm{cm}^{-1}$ indicates the occurrence of the thiol form. The results of quantum chemical calculations on the total energy of the transition complex in vacuum at $T=298 K$ and on the activation energy values of thione-thiol tautomeric transition between different conformations of the cluster models have shown a decrease in the activation barrier due to the grafting of thiourea groups on silica surface. The configurations of the transition complexes were also determined.
\end{abstract}

Keywords: mesoporous silica, thione-thiol tautomerism, IR spectroscopy, quantum chemical calculations, density functional theory method

\section{INTRODUCTION}

As well known, organic compounds containing thiourea group of $-\mathrm{NH}-\mathrm{C}(=\mathrm{S})-\mathrm{NH}-$ can exist in two tautomeric forms - thione and thiol, $-\mathrm{N}=\mathrm{C}(\mathrm{SH})-\mathrm{NH}-$. The shift of the tautomeric equilibrium is greatly influenced by the $\mathrm{pH}$ of the medium. In [1] it was shown that in an alkaline environment thione-thiol equilibrium shifts toward thiol form, and in neutral or slightly acidic towards thione one. This fact is confirmed in many studies, for example, in $[2,3]$. Existence of thionethiol tautomers also depends essentially on the nature of the solvent [4] since they consist in binary prototropic equilibrium. Previously [5] it was shown by semiempirical quantum chemical calculations that when weakly solvated with ethanol solution, thiol state became energetically more favorable, and an excess of ethanol is energetically more favorable for thione one. In [6] by the DFT (B3LYP / 6-31G (d, p)) simulation it was found that the presence of the hydration shell did affect the stability of discussed tautomeric forms. Prototropic tautomeric forms are difficult to isolate in the raw and to calculate their ratio [7] due to the considerable mobility of thione-thiol equilibrium and high rate interconversions. It should be added that the simple thiones are often unstable.
Since surface comprising ligands with thiourea groups are widely used for extraction of a number of metal ions from aqueous solutions [8], the question of coexistence of the two forms - before and after the sorption - is a topical. In the analysis of the IR spectra of the complexes formed in the surface layer of mesoporous silicas with the complexing group of $\left[\equiv \mathrm{Si}\left(\mathrm{CH}_{2}\right)_{3} \mathrm{NHC}(\mathrm{S}) \mathrm{NHC}_{2} \mathrm{H}_{5}\right]$, authors [9] found not one, but two absorption bands related to $-\mathrm{NH}-\mathrm{C}(\mathrm{S})-\mathrm{NH}-$ group taking part of the coordination complex. The authors suggested that due to formation of complexes on the surface of such sorbents, ligands exist in both thiol and thione forms. In the preparation of mesoporous sorbents with thiourea groups by templating method [10,11], deletion of the surfactant mesophase can be performed under various conditions (referring to the nature of the solvent, the acidity of the medium). This, in turn, may be associated with the appearance of tautomeric equilibrium on the support surface.

Since the above assumption was based on the analysis of the IR spectra, it should be noted that the thiourea moiety has no distinct characteristic absorption bands [11]. The absorption band at $\sim 1560 \mathrm{~cm}^{-1}$, common used for identifying this fragment, is caused by the contribution of several variations. The absorption bands, corresponding to the stretching vibrations of thione $[v(\mathrm{C}=\mathrm{S})]$ or thiol 
$[v(\mathrm{~S}-\mathrm{H})]$ form, have a low intensity. Therefore, at a low fixed content of the thiourea ligands, which is typical for the vast majority of sorbents, their identification is difficult in these absorption bands. It becomes even more problematic if the content of the thiol tautomer greatly reduced compared with thione. Finally, if the absorption band at $v(\mathrm{~S}-\mathrm{H})$ is in $\sim 2560 \mathrm{~cm}^{-1}$, where the absorption bands of other ligands rarely fixed, the absorption band $v(\mathrm{C}=\mathrm{S})$, located in the IR spectrum at $\sim 630 \mathrm{~cm}^{-1}$, often may be masked by other absorption bands. The aim of this work is: by quantum chemical calculations of the surface layer of thiourea-functionalized silica (HO) ${ }_{3} \mathrm{SiCH}_{2} \mathrm{NHC}(\mathrm{S}) \mathrm{NHCH}_{3}$, consideration the possibility of the existence and identification in IR spectroscopy absorption bands of the thione-thiol tautomerism.

As a model of ligand center, N, N'- dimethylthiourea molecule in various forms and the surface of the fragment $(\mathrm{HO})_{3} \mathrm{SiCH}_{2} \mathrm{NHC}(\mathrm{S}) \mathrm{NHCH}_{3}$ mimics used in the practice of trifunctional silane $\left(\mathrm{C}_{2} \mathrm{H}_{5} \mathrm{O}\right)_{3} \mathrm{Si}\left(\mathrm{CH}_{2}\right)_{3} \mathrm{NHC}(\mathrm{S}) \mathrm{NHC}_{2} \mathrm{H}_{5} \quad[10,11]$ were adopted.

\section{METHODS}

Quantum chemical calculations on the total energy of the optimized geometrical structures of thione and thiol states of $\mathrm{N}, \mathrm{N}$-dimethylthiourea and of the fragment $(\mathrm{HO})_{3} \mathrm{SiCH}_{2} \mathrm{NHC}(\mathrm{S}) \mathrm{NHCH}_{3}$ were performed using density functional theory method (DFT) [6] and the hybrid B3LYP potential [12] with basis set $6-31 \mathrm{G}(\mathrm{d}, \mathrm{p})$. The calculated vibrational frequencies in the IR spectra were used as scaled by a factor of 0.95 . Calculations were performed by means of the software package FireFly 8.0.0 [13, 14].

\section{RESULTS AND DISCUSSION}

Previously $[15,16]$ we designed a model for silica surface layer formed due to hydrolytic polycondensation of tri- and tetraalkoxysilanes. This silica surface considered bears grafted thiourea groups in thione form. In this work, as a model for thiourea complexing center, $\mathrm{N}, \mathrm{N}$-dimethylthiourea in thione $\left(1,1^{\prime}, 1^{\prime \prime}\right)$, thiol $\left(2,2^{\prime}\right)$ forms, transition state $\left(3,3^{\prime}\right)$ and a form where the proton is substituted with sodium cation ( $\left.3^{\prime \prime}\right)$ were adopted (see Fig. 1 and Table 1).

In addition, for a molecular fragment (HO) ${ }_{3} \mathrm{SiCH}_{2} \mathrm{NHC}(\mathrm{S}) \mathrm{NHCH}_{3}$, quantum chemical calculations were performed. Complexing site of this fragment is identical to that in the trifunctional silane $\left(\mathrm{C}_{2} \mathrm{H}_{5} \mathrm{O}\right)_{3} \mathrm{Si}\left(\mathrm{CH}_{2}\right)_{3} \mathrm{NHC}(\mathrm{S}) \mathrm{NHC}_{2} \mathrm{H}_{5}$ which is widely used to produce functionalized polysiloxane xerogels [9] and mesoporous silicas [11]. Thus, it considered thione $\left(4,4^{\prime}\right)$ and thiol form $\left(5,5^{\prime}, 5^{\prime \prime}\right)$, and transition state ( 6 and 6 ) (see Fig. 2 and Table 2).

As it can be seen from the figures and tables, the geometrical parameters of thione forms of $\mathrm{N}, \mathrm{N}$-dimethylthiourea and its analog $(\mathrm{HO})_{3} \mathrm{SiCH}_{2} \mathrm{NHC}(\mathrm{S}) \mathrm{NHCH}_{3}$ are practically identical (see 1 in the Table 1 and 4 in the Table 2). Some differences are observed in the bond lengths $R_{\mathrm{NC}}$ for 4 , but they are not significant and originate from the differences in the substituents at nitrogen atoms. A similar conclusion can be drawn considering the geometrical parameters of the thiol form (see 2 in the Table 1, $5^{\prime}$ and 5 in the Table 2). We note only that for the thiol forms, the difference in the carbon-nitrogen bond lengths $[-\mathrm{NH}-\mathrm{C}(\mathrm{SH})=\mathrm{N}-]$ is typical. It is caused by the fact that the sulfur atom has only one amide proton. When comparing the total energies of the considered molecular forms, in both cases, the energetically more favorable form is found to be the thione one (see Tables 1 and 2). It should be noted that the thiol forms 2 and 5 are the closest in energy to the thione forms 1 and 4, respectively. The difference between these forms is the orientation of the HS bond (see Tables 1 and 2). The rest of the forms $\left(1^{\prime}, 2^{\prime}, 4^{\prime}, 5^{\prime}\right)$ are energetically disadvantageous (as compared to the forms 1, 2, 4 and 5 , respectively) and do not need further treated. Forms 3, 6 and $6^{\prime}$ are the transition states in the thione-thiol tautomerism and considered in the discussion of the total energy diagram of the tautomeric transition.

After the geometry optimization of thione ( 1 and 4) and the thiol (2 and 5) forms of the $\mathrm{N}, \mathrm{N}$ '-dimethylthiourea and its analog $(\mathrm{HO})_{3} \mathrm{SiCH}_{2} \mathrm{NHC}(\mathrm{S}) \mathrm{NHCH}_{3}$, we were able to calculate the position of the bands in the IR spectra of these molecules and their relative intensity. Such data are of considerable interest in establishing a manner of coordination of the ligands to the metal. But this is especially important in the case, when there is no change in the coordination process of ligand binding, such as our case (thiourea monodentate ligand bound by a sulfur atom). However, changing the structure of the fragment occurs in coordination (tautomerism). The structure of this fragment includes the atom through which a ligand is coordinated to the metal. In this case it is a sulfur atom which is a neutral thione moiety $[-\mathrm{NH}-\mathrm{C}(=\mathrm{S})-\mathrm{NH}-]$ or is linked to a hydrogen 
atom in the thiol moiety $[-\mathrm{N}=\mathrm{C}(-\mathrm{SH})-\mathrm{NH}-]$. In the latter case appears azomethine bond $(-\mathrm{N}=\mathrm{C}<)$ which is usually easily fixed in the IR spectra in the "fingerprint" (above $1600 \mathrm{~cm}^{-1}$ ). First, we
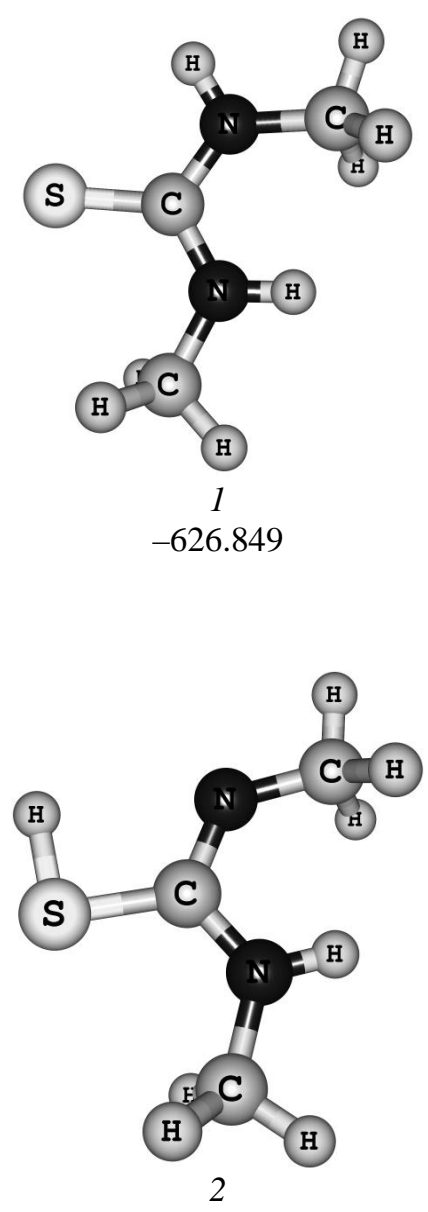

$-626.819$

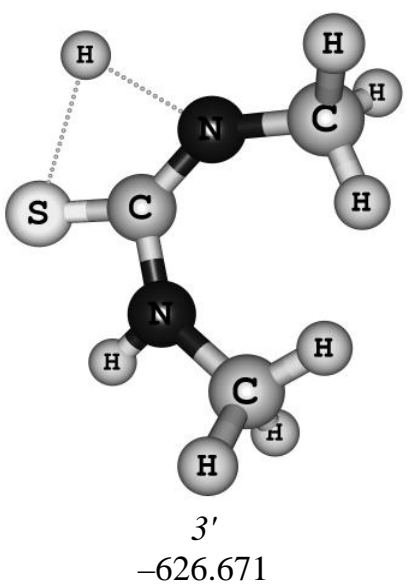

examined the position of the absorption bands in the IR spectra calculated thione (1) and thiol (2) forms of N, N'-dimethylthiourea (Table 3).
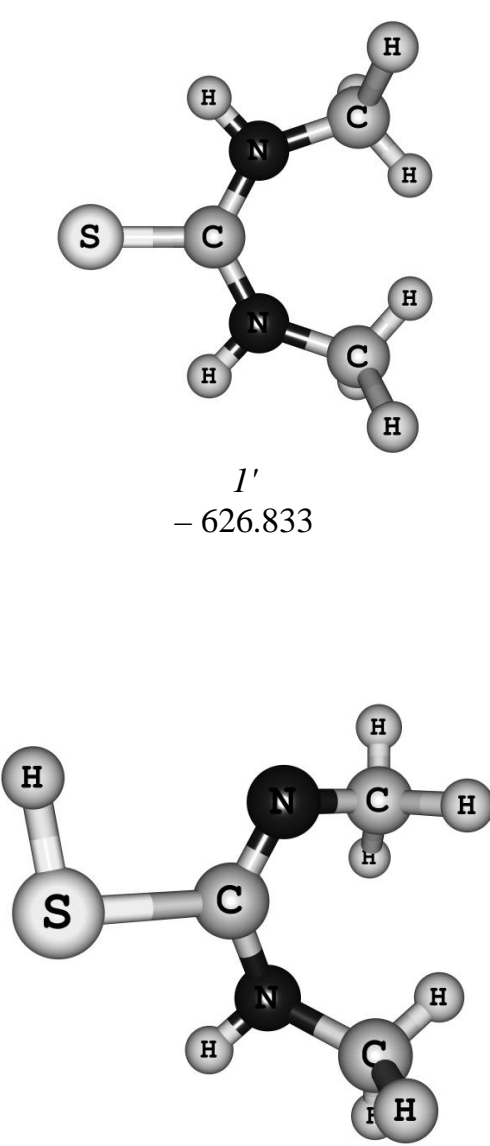

$2^{\prime}$

$-626.814$
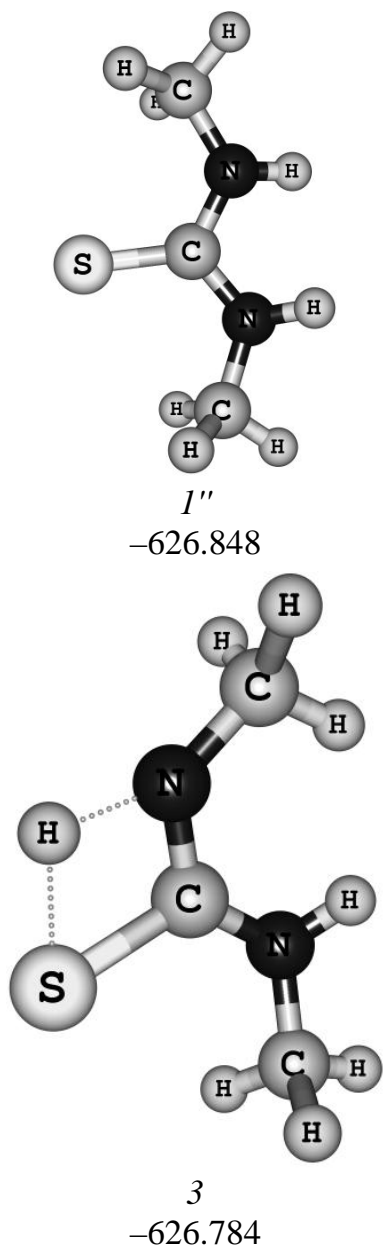

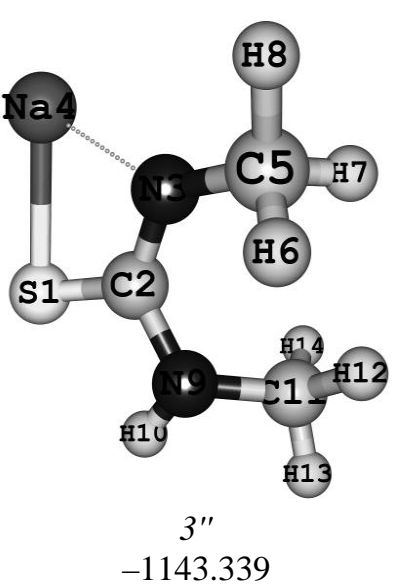

Fig. 1. The total energy calculation ( $E$, a.u.) of $\mathrm{N}, \mathrm{N}^{\prime}$-dimethylthiourea in various forms: $1,1^{\prime}, 1^{\prime \prime}-$ thione, $2,2^{\prime}-$ thiol, 3 , $3^{\prime}$ - transition state, $3^{\prime \prime}$ - a form where the proton is substituted with sodium cation 


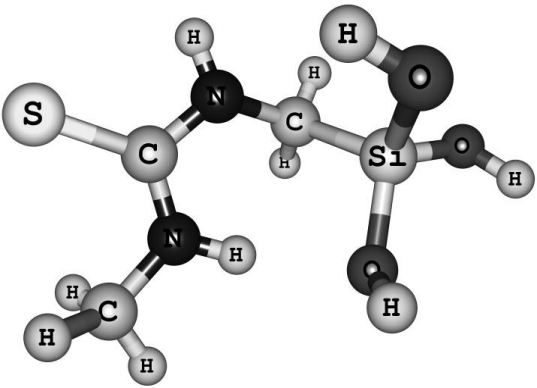

4 $-1143.3697$

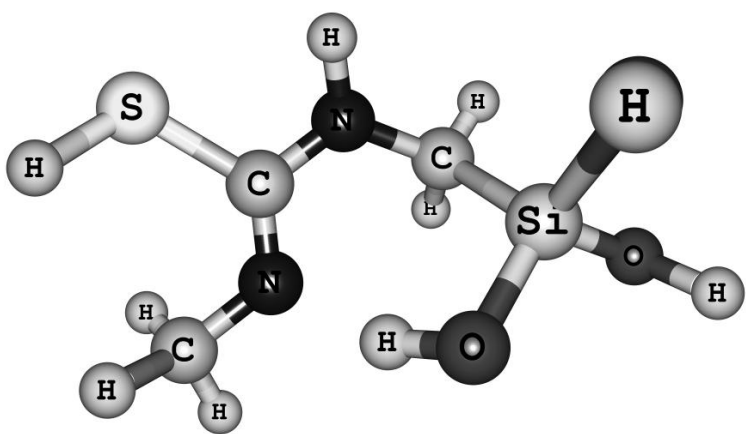

5

$-1143.3516$

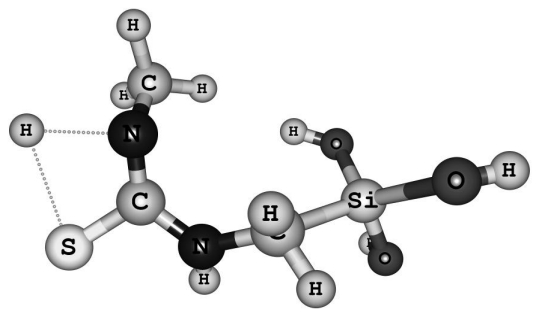

6

$-1143.1555$

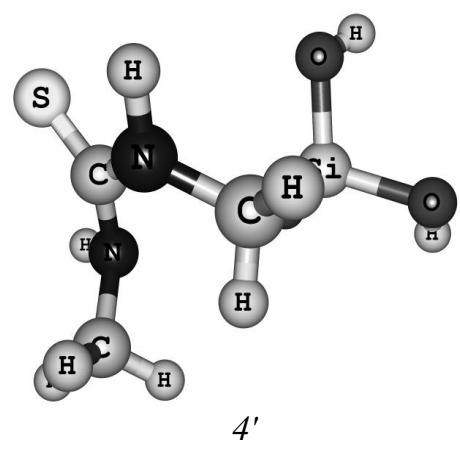

$-1143.3637$

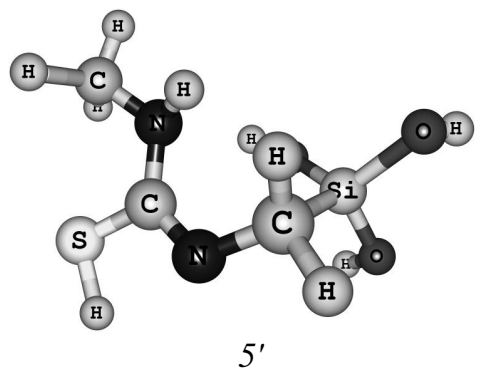

$-1143.3408$

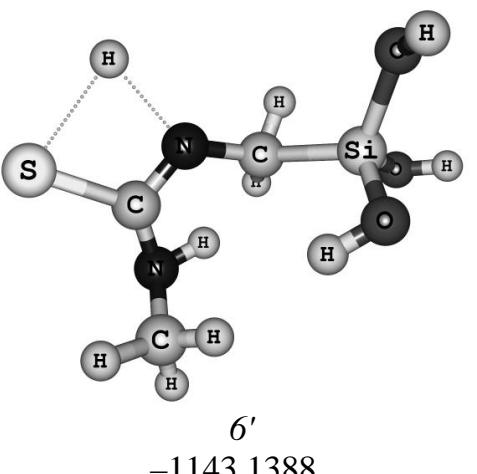

$-1143.1388$

Fig. 2. The calculated total energy $\left(E\right.$, a.u.) of molecular fragment $(\mathrm{HO})_{3} \mathrm{SiCH}_{2} \mathrm{NHC}(\mathrm{S}) \mathrm{NHCH}_{3}$ in various forms: $4,4^{\prime}-$ thione, 5, 5' - thiol and $6,6^{\prime}-$ transition state

Table 1. Parameters of the optimized geometrical structure of various forms of N, N'-dimethylthiourea

\begin{tabular}{lcccc}
\hline \multirow{2}{*}{ Parameters } & \multicolumn{4}{c}{ Forms } \\
\cline { 2 - 5 } & $\mathbf{1}$ & $\mathbf{2}$ & $\mathbf{3}$ & $\mathbf{3}^{\prime}$ \\
\hline$R_{\mathrm{SH}}\left(R_{\mathrm{SNa}}\right)$ & - & 1.345 & $2.297(\mathrm{SH})$ & $2.571(\mathrm{SNa})$ \\
$R_{\mathrm{CS}}$ & 1.688 & 1.808 & 1.636 & 1.773 \\
$R_{\mathrm{CN}}$ & 1.360 & $1.378,1.277$ & $1.359,1.390$ & $1.396,1.311$ \\
$R_{\mathrm{NC}}$ & 1.454 & $1.455,1.454$ & $1.456,1.456$ & $1.456,1.465$ \\
$R_{\mathrm{CH}}$ & $1.092-1.099$ & $1.094-1.104$ & $1.083-1.10$ & $1.09-1.10$ \\
$R_{\mathrm{NH}}, R_{\mathrm{NNa}}$ & 1.008 & 1.009 & $1.035,1.834^{*}$ & $1.012,2.254^{*}$ \\
$<\mathrm{HSC}(<\mathrm{NaSC})$ & - & 92.22 & 59.35 & 75.03 \\
$<\mathrm{SCN}$ & 120.85 & $114.10,118.59$ & $103.29,136.65$ & $121.50,114.37$ \\
$<\mathrm{NCN}$ & 115.90 & 127.28 & 120.06 & 124.11 \\
$<\mathrm{CNH},<\mathrm{CNNa}$ & 119.03 & 114.08 & $115.01,77.39 *$ & $109.21,95.80^{*}$ \\
$<\mathrm{NCH}$ & $108.08-112.06$ & $108.44-113.33$ & $110.20-112.81$ & $107.82-113.19$ \\
\hline
\end{tabular}

* Italicized values for $R_{\mathrm{NH}},<\mathrm{CNH}$ to 3 and for $R_{\mathrm{NNa}},<\mathrm{CNNa}$ to $3^{\prime}$ 
Table 2. Parameters of the optimized geometry of the molecular fragment $(\mathrm{HO})_{3} \mathrm{SiCH}_{2} \mathrm{NHC}(\mathrm{S}) \mathrm{NHCH}_{3}$ in various forms

\begin{tabular}{lcccc}
\hline \multirow{2}{*}{ Parameters } & \multicolumn{4}{c}{ Forms } \\
\cline { 2 - 5 } & $\mathbf{4}$ & $\mathbf{5}$ & $\mathbf{6}$ & $\mathbf{6}^{\prime}$ \\
\hline$R_{\mathrm{SH}}$ & - & 1.347 & 2.291 & 2.373 \\
$R_{\mathrm{CS}}$ & 1.687 & 1.824 & 1.825 & 1.825 \\
$R_{\mathrm{CN}}$ & $1.352,1.379$ & $1.282,1.365$ & $1.282,1.362$ & $1.282,1.362$ \\
$R_{\mathrm{NC}}$ & $1.379,1.455$ & $1.452,1.471$ & $1.469,1.282$ & $1.282,1.469$ \\
$R_{\mathrm{CH}}$ & $1.095-1.092$ & $1.093-1.097$ & $1.094-1.099$ & $1.093-1.099$ \\
$R_{\mathrm{NH}}$ & $1.015,1.011$ & 1.01 & 1.009 & 1.009 \\
$R_{\mathrm{SiO}}$ & $1.649-1.677$ & $1.664-1.661$ & $1.648-1.668$ & $1.648-1.668$ \\
$<\mathrm{HSC}$ & - & 95.97 & 72.021 & 67.417 \\
$<\mathrm{SCN}$ & $119.94,123.91$ & $110.14,126.76$ & $110.30,124.24$ & $110.30,124.24$ \\
$<\mathrm{NCN}$ & 116.14 & 122.96 & 124.82 & 124.82 \\
$<\mathrm{NCSi}$ & 112.44 & 115.03 & 115.81 & 115.81 \\
$<\mathrm{CSiO}$ & $106.77-110.82$ & 109.82 & $102.85-112.22$ & $102.85-112.22$ \\
$<\mathrm{CNH}$ & $110.60,119.20$ & 115.79 & 117.80 & 117.80 \\
$<\mathrm{NCH}$ & $108.02-111.80$ & 107.07 & $107.38-112.97$ & $108.09-112.97$ \\
$<\mathrm{SiCH}$ & $107.37,111.55$ & $107.73-110.25$ & $108.34,110.12$ & $108.34-110.12$ \\
$<\mathrm{OSiO}$ & $108.58-111.49$ & $107.73-115.25$ & $107.85-114.90$ & $107.85-114.90$ \\
$<\mathrm{SiOH}$ & $114.21-117.66$ & $112.56-115.33$ & $109.12-115.88$ & $109.12-115.88$ \\
\hline
\end{tabular}

Table 3. Calculated (B3LYP/6-31G (d,p) IR spectra of N, N'-dimethylthiourea

\begin{tabular}{|c|c|c|c|c|c|}
\hline \multicolumn{3}{|c|}{$\underline{1 \text { (thione) }}$} & \multicolumn{3}{|c|}{$\underline{2 \text { (thiol) }}$} \\
\hline $\begin{array}{l}\text { Frequency, } \\
\text { cm }^{-1}\end{array}$ & Intensity & PED* & $\begin{array}{l}\text { Frequency, } \\
\text { cm }^{-1}\end{array}$ & Intensity & PED* \\
\hline 1 & 2 & 3 & 4 & 5 & 6 \\
\hline & & & 74 & 0.04 & $\rho_{\mathrm{CNCS}}, \rho_{\mathrm{CH}_{3}}$ \\
\hline 120 & 0.03 & $\rho_{\mathrm{CH}_{3}}$ & 131 & 0.00 & $\rho_{\mathrm{CNCS}}, \rho_{\mathrm{CH}_{3}}$ \\
\hline & & & 165 & 0.19 & $\rho_{\mathrm{SCNC}}, \rho_{\mathrm{HSCN}}, \rho_{\mathrm{HCNC}}$ \\
\hline 176 & 0.32 & $\rho_{\mathrm{CH}_{3}}$ & & & \\
\hline & & & $\begin{array}{l}210 \\
226\end{array}$ & $\begin{array}{l}0.04 \\
0.01\end{array}$ & $\begin{array}{c}\rho_{\mathrm{SCNC}} \\
\delta^{\text {as }}{ }_{\mathrm{NCS}}, \delta_{\mathrm{CNC}}^{\mathrm{s}}\end{array}$ \\
\hline 232 & 0.02 & $\begin{array}{c}\delta_{\mathrm{NCS},}^{\mathrm{s}} \delta^{\mathrm{as}}{ }_{\mathrm{CNC}} \\
\delta^{\mathrm{as}} \mathrm{CH}_{3}\end{array}$ & 264 & 0.10 & $\delta_{\mathrm{NCS}}^{\mathrm{s}}, \delta^{\mathrm{as}}{ }_{\mathrm{CNC}}, \rho_{\mathrm{CH}_{3}}$ \\
\hline 275 & 0.17 & $\delta^{\mathrm{as}} \mathrm{NCS}, \delta_{\mathrm{CNC}}^{\mathrm{s}}$ & $\begin{array}{l}308 \\
377\end{array}$ & $\begin{array}{l}0.17 \\
1.48\end{array}$ & $\begin{array}{c}\delta_{\mathrm{NCS}}^{\mathrm{s}}, \delta_{\mathrm{CNC}}^{\mathrm{s}}, \rho_{\mathrm{HSCN}}, \rho_{\mathrm{HNCS}} \\
\delta_{\mathrm{CNC}}^{\mathrm{s}} \delta^{\text {as }}{ }_{\mathrm{NCS}} \rho_{\mathrm{HNCS}}\end{array}$ \\
\hline 319 & 2.27 & $\rho_{\mathrm{CH}_{3}}$ & 430 & 0.38 & $v_{\mathrm{CS}}, \delta_{\mathrm{NCS}}^{\mathrm{as}}, \delta_{\mathrm{CNC},}^{\mathrm{as}} \rho_{\mathrm{HNCS}}$ \\
\hline 442 & 0.45 & $\begin{array}{c}v_{\mathrm{CS}}, v_{\mathrm{NC}}, \delta^{\mathrm{as}}{ }_{\mathrm{CNC}} \\
\delta^{\text {as }}{ }_{\mathrm{NCS}}, \rho_{\mathrm{HNCS}}\end{array}$ & & & \\
\hline 457 & 0.61 & $\begin{array}{c}v_{\mathrm{CS}}, v_{\mathrm{NC}}, \delta^{\mathrm{as}}{ }_{\mathrm{CNC}} \\
\delta_{\mathrm{NCS}}^{\mathrm{s}}, \delta^{\mathrm{as}}{ }_{\mathrm{NCS}}, \rho_{\mathrm{HNCS}}\end{array}$ & & & \\
\hline 570 & 0.19 & $v_{\mathrm{CS}}, v_{\mathrm{NC}}, \delta_{\mathrm{NCS}}^{\mathrm{s}}, \delta^{\mathrm{as}}{ }_{\mathrm{NCS}}$ & 518 & 0.74 & $v_{\mathrm{CS}}, \delta_{\mathrm{NCS}}^{\mathrm{s}}, \delta_{\mathrm{CNC},}^{\mathrm{as}} \rho_{\mathrm{HNCS}}$ \\
\hline 638 & 0.17 & $\rho_{\mathrm{HNCS}}$ & $\begin{array}{l}647 \\
714\end{array}$ & $\begin{array}{l}0.57 \\
0.37\end{array}$ & $\begin{array}{c}\rho_{\mathrm{HNCS}} \\
v_{\mathrm{CS}}, v_{\mathrm{CNC},} \delta^{\mathrm{as}}{ }_{\mathrm{CNC}}\end{array}$ \\
\hline 741 & 0.14 & $v^{\mathrm{as}}{ }_{\mathrm{NCN}}, v^{\mathrm{as}}{ }_{\mathrm{CNC}}, \delta^{\mathrm{as}}{ }_{\mathrm{CNC}}$ & 906 & 0.75 & $\delta_{\mathrm{CSH},} v_{\mathrm{NCN}}^{\mathrm{s}}, \delta^{\mathrm{as}}{ }_{\mathrm{CNC}}, \delta^{\mathrm{as}}{ }_{\mathrm{NCS}}, \rho_{\mathrm{HNCS}}$ \\
\hline 1050 & 0.05 & $v_{\mathrm{CS}}, v_{\mathrm{NC}}, v_{\mathrm{HC}}, \delta_{\mathrm{HCN}}$ & $\begin{array}{l}1008 \\
1076\end{array}$ & $\begin{array}{l}0.23 \\
1.03\end{array}$ & $\begin{array}{c}v_{\mathrm{CS}}, v_{\mathrm{CN}} \\
\delta^{\text {as }}{ }_{\mathrm{NCS}}, v_{\mathrm{CN}}, \delta_{\mathrm{HCN}}^{\mathrm{s}}\end{array}$ \\
\hline 1065 & 1.15 & $v_{\mathrm{CS},} v_{\mathrm{CNC},}^{\mathrm{s}} \delta_{\mathrm{NCS}}^{\mathrm{s}}$ & & & \\
\hline 1147 & 0.00 & $\delta_{\mathrm{HCN}}^{\mathrm{as}}, \rho_{\mathrm{HCNC}}$ & $\begin{array}{l}1106 \\
1143 \\
1156\end{array}$ & $\begin{array}{l}0.02 \\
0.44 \\
0.13\end{array}$ & $\begin{array}{c}\delta^{\mathrm{as}}{ }_{\mathrm{HCN},} \delta^{\mathrm{as}}{ }_{\mathrm{CNC},} \rho_{\mathrm{HCNC}} \\
\delta^{\mathrm{as}}{ }_{\mathrm{HCN}} \\
\delta^{\mathrm{as}}{ }_{\mathrm{HCN}}\end{array}$ \\
\hline 1155 & 0.03 & $\delta_{\text {HCN }}^{\text {as }}$ & 1216 & 0.06 & $v_{\mathrm{CN}}, \delta^{\mathrm{as}}{ }_{\mathrm{HCN}}$ \\
\hline 1181 & 0.01 & $v^{\mathrm{as}}{ }_{\mathrm{CNC}}, \delta^{\mathrm{as}}{ }_{\mathrm{HCN}}$ & & & \\
\hline
\end{tabular}




\begin{tabular}{|c|c|c|c|c|c|}
\hline 1 & 2 & 3 & 4 & 5 & 6 \\
\hline 1215 & 0.28 & $\rho_{\mathrm{HCNC},} \delta^{\text {as }}{ }_{\mathrm{HCN}}$ & & & \\
\hline 1281 & 4.00 & $v^{\text {as }}{ }^{\text {and }}, \delta_{\text {HNC, }}^{\text {as }}$ & & & \\
\hline 1371 & 222 & & 1301 & 3.44 & $v_{\mathrm{CN}}, \delta^{\mathrm{as}}{ }_{\mathrm{HNC},} \delta^{\mathrm{as}}{ }_{\mathrm{NCS}}, \delta_{\mathrm{HSC},}^{\mathrm{as}} \delta_{\mathrm{CNC},}^{\mathrm{as}}$ \\
\hline & & & 1440 & 1.48 & $\delta_{\mathrm{HCN},}^{\mathrm{s}} v_{\mathrm{CS}}$ \\
\hline 1467 & 0.25 & $\delta_{\mathrm{NCN}}^{\mathrm{s}}, \delta_{\mathrm{NCN}}^{\mathrm{s}}, \delta_{\mathrm{HCN}}^{\mathrm{s}}$ & & & \\
\hline 1483 & 0.05 & $\delta_{\mathrm{HNC}}^{\mathrm{as}}, \delta^{\mathrm{as}}{ }_{\mathrm{CNC}}, \rho_{\mathrm{HCNS}}$ & 1473 & 0.37 & $\delta^{\text {as }} \mathrm{HNC}, \rho_{\mathrm{HCNC}}$ \\
\hline 1484 & 0.16 & $\rho_{\mathrm{HCNC}}$ & 1482 & 0.20 & $\rho_{\mathrm{HCNC}}, \delta_{\mathrm{HNC}}^{\mathrm{s}}$ \\
\hline 1498 & 0.17 & $\rho_{\mathrm{HCNC},} \delta^{\mathrm{as}}{ }_{\mathrm{HNC}}$ & 1504 & 0.31 & $\rho_{\mathrm{HCNC}}, \delta_{\mathrm{HNC}}^{\mathrm{s}}$ \\
\hline & & & 1512 & 0.04 & $v_{\mathrm{CS}}, \delta_{\mathrm{HCN}}^{\mathrm{s}}, v_{\mathrm{CN}}^{\mathrm{as}}$ \\
\hline & & & 1521 & 0.13 & $\rho_{\mathrm{HCNC}}$ \\
\hline 1520 & 0.15 & $\rho_{\mathrm{HCNC},} \delta^{\text {as }}{ }_{\mathrm{HNC}}$ & 1533 & 0.59 & $\begin{array}{c}\delta^{\mathrm{as}}{ }_{\mathrm{HCN},} \delta^{\mathrm{as}}{ }_{\mathrm{HCN}}, \\
\rho_{\mathrm{HCNC}}\end{array}$ \\
\hline 1531 & 0.56 & $\delta^{\mathrm{as}}{ }_{\mathrm{HNC}}, \delta^{\mathrm{as}}{ }_{\mathrm{HCN}}, \rho_{\mathrm{HCNC}}$ & & & \\
\hline 1544 & 5.28 & $v_{\mathrm{CS}}, v^{\mathrm{as}}{ }_{\mathrm{NCN}}, \delta^{\mathrm{as}}{ }_{\mathrm{HNC}}$ & & & \\
\hline 1586 & 6.80 & $v_{\mathrm{NCN}}^{\mathrm{s}}, \delta^{\text {as }}{ }_{\mathrm{HNC}}$ & $\begin{array}{l}1714 \\
2718 \\
2953\end{array}$ & $\begin{array}{c}9.76 \\
0.002 \\
1.84\end{array}$ & $\begin{array}{c}v_{\mathrm{CS},}, v_{\mathrm{NCN},}^{\mathrm{s}} \delta^{\mathrm{as}}{ }_{\mathrm{HCN}} \\
v_{\mathrm{SH}} \\
v^{\mathrm{as}} \mathrm{CH}_{2}\end{array}$ \\
\hline 3004 & 1.30 & $v^{\text {as }} \mathrm{CH}_{2}$ & 3010 & 1.28 & $v^{\text {as }}{ }_{\mathrm{CH}_{2}}$ \\
\hline 3056 & 0.91 & $v_{\mathrm{CH} 2,}^{\mathrm{s}} v_{\mathrm{CH} 2}^{\mathrm{as}}$ & 3016 & 1.38 & $v_{\mathrm{CH}_{2}}^{\mathrm{s}}$ \\
\hline 3062 & 0.90 & $v^{\text {as }} \mathrm{CH}_{2}$ & 3100 & 0.82 & $v^{\mathrm{s}} \mathrm{CH}_{2}$ \\
\hline 3127 & 0.37 & $v^{\mathrm{s}} \mathrm{CH}_{2}^{2}$ & 3108 & 0.43 & $v^{\text {as }}{ }_{\mathrm{CH}_{2}}, v^{\mathrm{s}} \mathrm{CH}_{2}$ \\
\hline 3134 & 0.25 & $v^{\mathrm{s}} \mathrm{CH}_{2}$ & 3150 & 0.45 & $v^{s^{2}} \mathrm{CH}_{2}$ \\
\hline 3154 & 0.51 & $v^{\mathrm{s}} \mathrm{CH}_{2}^{2}$ & 3626 & 0.45 & $v_{\mathrm{NH}}^{2}$ \\
\hline 3627 & 0.46 & $v_{\mathrm{NH}}$ & & & \\
\hline
\end{tabular}

"Potential energy distribution (PED) calculated from B3LYP/6-31G (d, p) method, contributions are listed than $10 \%$ and in descending order

In the Table 3 it is shown that the IR spectra thione and thiol forms of $\mathrm{N}, \mathrm{N}$ '-dimethylthiourea significantly differ in the position of the four absorption bands. Thus, for the thione form absorption bands at 1544 and $1586 \mathrm{~cm}^{-1}$ are fixed (can be attributed to $v_{\mathrm{CS}}, v_{\mathrm{NCN}}^{\mathrm{as}} \delta_{\mathrm{HNC}}^{\mathrm{as}} v_{\mathrm{CNC}}^{\mathrm{s}}, \delta_{\mathrm{HCN}}^{\mathrm{as}}$, $\rho_{\mathrm{HCNC}}$ and $v_{\mathrm{NCN}}^{\mathrm{s}} \delta^{\text {as }}{ }_{\mathrm{HNC}}$ ) and they are absent in the IR spectrum of its thiol form. IR spectrum of thiol form contains two bands at 1714 and $2718 \mathrm{~cm}^{-1}$ which are absent in the IR spectrum of thione form. They can be attributed to the vibrations $v_{\mathrm{NC}}, \delta_{\mathrm{NCN}}^{\mathrm{s}}, \delta^{\text {as }}{ }_{\mathrm{HCN}}$ and $v_{\mathrm{HS}}$ respectively. Since the thiol proton will be replaced by cations of the metal, the characteristic absorption bands should be considered an absorption band at $1586 \mathrm{~cm}^{-1}$ in the case of the thione forms and $1714 \mathrm{~cm}^{-1}$ in the case of the thiol form.

A similar conclusion can be made after the analysis of Table 4 which shows the position of the absorption bands in the calculated IR spectra of molecular fragments $(\mathrm{HO})_{3} \mathrm{SiCH}_{2} \mathrm{NHC}(\mathrm{S}) \mathrm{NHCH}_{3}$ in thione and thiol forms. In this case the characteristic absorption bands should be used a band at $1607 \mathrm{~cm}^{-1}\left(v_{\mathrm{NCN}}^{\mathrm{s}}\right)$ for the thione form and $1707 \mathrm{~cm}^{-1}\left(v_{\mathrm{NCN}}^{\mathrm{s}}\right)$ in case of the thiol one.

The activation energy of the transition between the thione 1 and 2 states of thiol molecule of $\mathrm{N}, \mathrm{N}$-dimethylthiourea is $E^{\mathrm{A}}=69.05 \mathrm{~kJ} / \mathrm{mol}$. Accordingly, for a molecule fragment simulating the silica surface with a functional group [-NHC(S)NH-], this value is $E^{\mathrm{A}}=47.52 \mathrm{~kJ} / \mathrm{mol}$ in the case of transition $4 \rightarrow 5$ or $E^{\mathrm{A}}=75.88 \mathrm{~kJ} / \mathrm{mol}$ in the case of transition $4 \rightarrow 5^{\prime}$ (Fig. 3). Comparing the calculated values of the activation energy, we can conclude that in the case of a fragment of the surface (HO) ${ }_{3} \mathrm{SiCH}_{2} \mathrm{NHC}(\mathrm{S}) \mathrm{NHCH}_{3}$ activation barrier of thione-thiol tautomeric transition decreases. Also, one can conclude that the transition of the hydrogen atom to sulfur atom is likely to be 
involved nitrogen, maximally distant from the surface of silica (condition 6, Fig. 2 and 3), since the activation energy in this case is smaller than in the state $6^{\prime}$.

Table. 4. The calculated IR spectra of the fragment $(\mathrm{HO})_{3} \mathrm{SiCH}_{2} \mathrm{NHC}(\mathrm{S}) \mathrm{NHCH}_{3}$ in thione and thiol forms

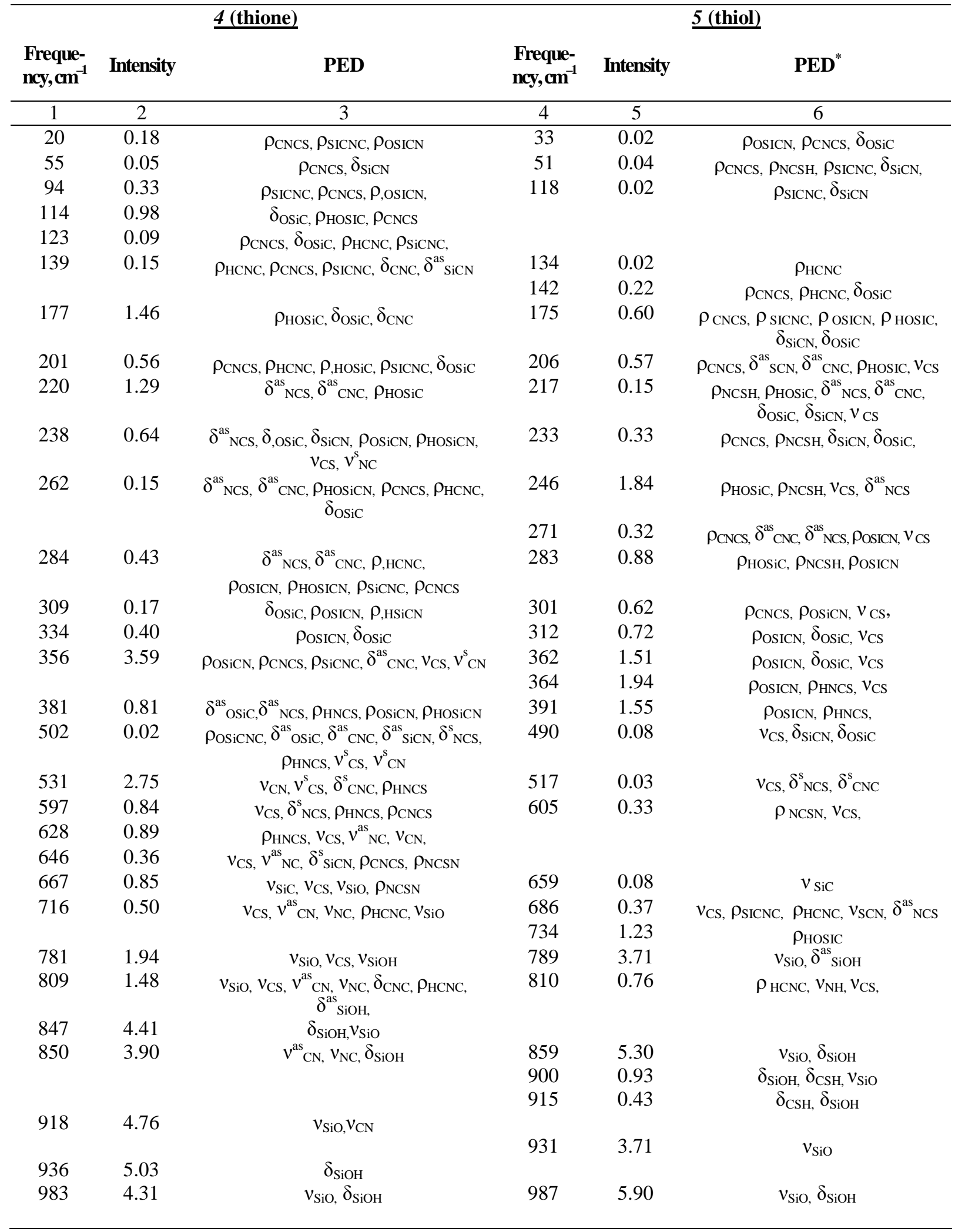


Thione-thiol tautomerism of thiourea ligands on silica surface

\begin{tabular}{|c|c|c|c|c|c|}
\hline 1 & 2 & 3 & 4 & 5 & 6 \\
\hline 1032 & 0.28 & $v_{\mathrm{CS}}, v_{\mathrm{NC}}$ & 1024 & 1.90 & $v_{\mathrm{CS}}, v_{\mathrm{CN}}, v_{\mathrm{NC}}, \delta_{\mathrm{NCS}}^{\mathrm{s}}$ \\
\hline \multirow[t]{3}{*}{1092} & 1.01 & $v_{\mathrm{NC}}, v_{\mathrm{CS}}, v_{\mathrm{CN}}, \delta_{\mathrm{HCN}} \delta^{\mathrm{as}}{ }_{\mathrm{NCS}}$ & 1075 & 1.80 & $v_{\mathrm{CN},}, v_{\mathrm{NC}}, \delta_{\mathrm{HCN}}^{\mathrm{as}}$ \\
\hline & & & 1125 & 0.09 & $\delta^{\text {as }}{ }_{\text {HCN },} \delta^{\text {as }}{ }_{\text {NCS }}$ \\
\hline & & & 1150 & 1.03 & $\delta_{\mathrm{HCN}}^{\text {as }} \delta_{\mathrm{SiOH},} \delta^{\text {as }}{ }_{\mathrm{NCS}}, v_{\mathrm{CN}}$ \\
\hline \multirow[t]{2}{*}{1152} & 0.13 & $\rho_{\mathrm{HCNC}}, \delta_{\mathrm{HCN}}$ & & & \\
\hline & & & 1164 & 2.32 & $\delta_{\mathrm{SiOH}}, \delta_{\mathrm{HCN}}$ \\
\hline 1182 & 0.24 & $v_{\mathrm{CN}}, \delta^{\mathrm{as}}{ }_{\mathrm{NCS}}, \delta_{\mathrm{HCN}}$ & & & \\
\hline 1252 & 1.29 & $v_{\mathrm{NC}}, v_{\mathrm{CS}}, v_{\mathrm{CN}}, \delta_{\mathrm{HNC}}, \delta_{\mathrm{HCN}}$ & & & \\
\hline 1268 & 2.88 & $v_{\mathrm{CS}}, v_{\mathrm{NC}}, v_{\mathrm{CN}}, \delta_{\mathrm{NCS}}, \delta_{\mathrm{CNC}}^{\mathrm{s}} \delta_{\mathrm{HNC}} \delta_{\mathrm{HCN}}$ & 1273 & 1.30 & 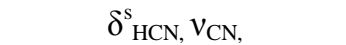 \\
\hline \multirow[t]{2}{*}{1298} & 0.36 & $\delta_{\mathrm{HCN},} v_{\mathrm{CN}}, \rho_{\mathrm{HCNC}}$ & 1282 & 0.38 & $v_{\mathrm{CN}}, v_{\mathrm{NC}}, \delta_{\mathrm{HCN}}^{\mathrm{as}}, \delta_{\mathrm{HNC}}$ \\
\hline & & & 1313 & 0.84 & $\delta_{\mathrm{HCN}}^{\mathrm{as}}, \rho_{\mathrm{HCNC}}, v_{\mathrm{SIC}}, \delta_{\mathrm{HNC}}$ \\
\hline \multirow[t]{3}{*}{1400} & 2.85 & $v_{\mathrm{NC}}, v_{\mathrm{CN}}, \delta_{\mathrm{CNC}}^{\mathrm{s}} \delta_{\mathrm{HCN}}, \delta_{\mathrm{HNC}} \rho_{\mathrm{HCNC}}$ & & & \\
\hline & & & 1453 & 1.04 & $\delta^{\mathrm{as}}{ }_{\mathrm{NCH} 3}, \rho_{\mathrm{HCNC}}$ \\
\hline & & & 1458 & 0.35 & $\delta^{\mathrm{as}}{ }_{\mathrm{NCH}}, \delta_{\mathrm{HCN}}, \rho_{\mathrm{HCNC}}$ \\
\hline 1461 & 0.49 & $\delta_{\mathrm{HCN},} \delta_{\mathrm{HNC},} \rho_{\mathrm{HCNC}}$ & & & \\
\hline 1479 & 1.20 & $\delta_{\mathrm{HCN}}, \rho_{\mathrm{HCNC},} v_{\mathrm{CN}}$ & & & \\
\hline \multirow[t]{2}{*}{1487} & 0.25 & $\rho_{\mathrm{HCNC},} \delta_{\mathrm{HCN}}$ & & & \\
\hline & & & 1504 & 0.15 & $\delta^{\mathrm{as}}{ }_{\mathrm{NCH} 3}, \rho_{\mathrm{HCNC}}, \delta_{\mathrm{HNC}}$ \\
\hline \multirow[t]{2}{*}{1518} & 0.09 & $v_{\mathrm{NC}}, \delta_{\mathrm{HCN}}, \rho_{\mathrm{HCNC}}$ & 1518 & 0.51 & $\rho_{\mathrm{HCNC}}, \delta_{\mathrm{HCN}}, \delta_{\mathrm{HNC}}$ \\
\hline & & & 1527 & 3.00 & $\delta_{\mathrm{HNC}} v_{\mathrm{NCN}}^{s}$ \\
\hline 1533 & 3.70 & $v_{\mathrm{CS}}, v_{\mathrm{NC}}, \delta_{\mathrm{HCN}}, \rho_{\mathrm{HCNC}}, \delta_{\mathrm{HNC}}, \delta_{\mathrm{NCS}}$ & & & \\
\hline \multirow[t]{3}{*}{1607} & 4.42 & $v_{\mathrm{NCN},}^{\mathrm{s}} \delta_{\mathrm{CNC},} v_{\mathrm{CN}}^{\mathrm{s}}, \delta_{\mathrm{HCN},} \rho_{\mathrm{HNCS}}$ & & & \\
\hline & & & 1707 & 7.84 & $v_{\mathrm{NCN}}^{\mathrm{s}}$ \\
\hline & & & 2708 & 0.12 & $v_{\mathrm{SH}}$ \\
\hline 3018 & 0.41 & $v^{\text {as }} \mathrm{CH}_{2}$ & 3013 & 1.47 & $v^{\mathrm{as}} \mathrm{CH}_{3}$ \\
\hline 3052 & 1.02 & $v^{\text {as }} \mathrm{CH}_{3}$ & 3049 & 0.30 & $v^{\text {as }} \mathrm{CH}_{2}$ \\
\hline 3116 & 0.12 & $v^{\mathrm{s}} \mathrm{CH}_{2}^{3}$ & 3071 & 0.81 & $v^{\mathrm{s}} \mathrm{CH}_{3}$ \\
\hline 3121 & 0.47 & $v^{\mathrm{s}} \mathrm{CH}_{3}^{2}$ & 3103 & 0.53 & $v^{\mathrm{s}} \mathrm{CH}_{3}$ \\
\hline 3146 & 0.23 & $v^{\mathrm{s}} \mathrm{CH}_{3}$ & 3119 & 0.22 & $v_{\mathrm{CH}_{2}}^{\mathrm{s}}$ \\
\hline 3518 & 4.76 & $v_{\mathrm{NH}}$ & 3403 & 21.47 & $v_{\mathrm{OH}}$ \\
\hline 3640 & 1.00 & $v_{\mathrm{NH}}$ & & & \\
\hline 3862 & 1.89 & $v_{\mathrm{OH}}$ & 3673 & 1.09 & $v_{\mathrm{NH}}$ \\
\hline 3874 & 1.82 & $v_{\mathrm{OH}}$ & 3867 & 1.45 & $v_{\mathrm{OH}}$ \\
\hline 3892 & 1.92 & $v_{\mathrm{OH}}$ & 3899 & 1.46 & $v_{\mathrm{OH}}$ \\
\hline
\end{tabular}
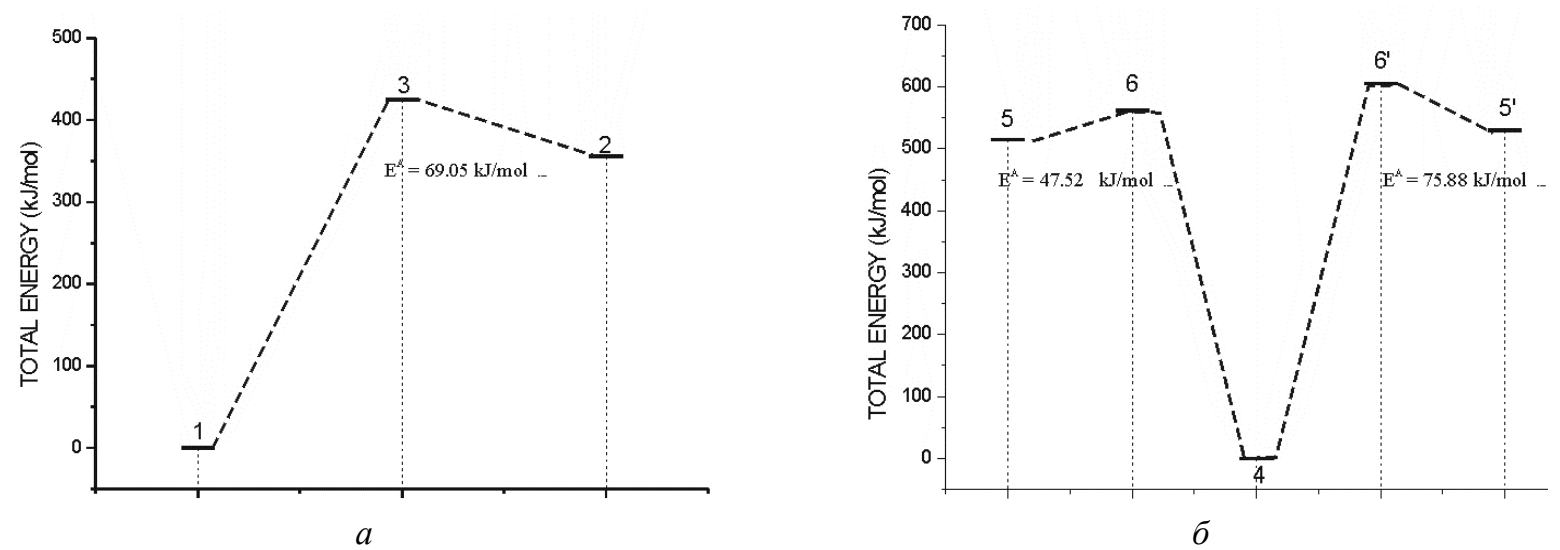

Fig. 3. Energy tautomeric transition diagram of $\mathrm{N}, \mathrm{N}^{\prime}$-dimethylthiourea $(a)$ and fragment cluster silica with the functional group [-NHC(S)NH-] (b) 


\section{CONCLUSIONS}

The possibility of the existence and identification of thione-thiol tautomerism on the surface of silica sorbents was considered. As the model were $\mathrm{N}, \mathrm{N}^{\prime}$ - dimethylthiourea molecule and the surface fragment $(\mathrm{HO})_{3} \mathrm{SiCH}_{2} \mathrm{NHC}(\mathrm{S}) \mathrm{NHCH}_{3}$. Based on the results of quantum chemical calculations, the interpretation of their IR spectrum was given. It has been shown that the presence of absorption bands at $1586 \mathrm{~cm}^{-1}$ (or $1607 \mathrm{~cm}^{-1}$ in the case of fragment surface) indicates the presence thione forms $[-\mathrm{NH}-\mathrm{C}(=\mathrm{S})-\mathrm{NH}-]$ and the appearance of an intense absorption band in 1714 (1707) $\mathrm{cm}^{-1}$ indicates the existence of a thiol form
$[-\mathrm{N}=\mathrm{C}(-\mathrm{SH})-\mathrm{NH}-]$. It has been found that for the fragment (HO) $)_{3} \mathrm{SiCH}_{2} \mathrm{NHC}(\mathrm{S}) \mathrm{NHCH}_{3}$ the activation barrier thione-thiol tautomeric transition decreases. Furthermore, the transition of the hydrogen atom to sulfur atom is likely to be involved nitrogen, maximum distance from the silica surface.

\section{ACKNOWLEDGEMENT}

The authors are grateful for the State Target Scientific and Technical Program of NAS of Ukraine «Nanotechnologies and Nanomaterials» (Project No 6.22.5.42).

\title{
Тіон-тіольна таутомерія тіосечовинних лігандів на поверхні кремнезему
}

\author{
О.В. Смірнова, А.Г. Гребенюк, Г.І. Назарчук, Ю.Л. Зуб
}

Інститут хімії поверхні ім. О.О. Чуйка Наџіональної академії наук України вул. Генерала Наумова, 17, Київ, 03164, Украӥна, оsтirnova@isc.gov.иа

Квантовохімічні розрахунки ІЧ-спектрів тіонної та тіольної форм $N, N^{\prime}$-диметилтіосечовини $i$ фрагмента поверхні кремнезему складу $(\mathrm{HO})_{3} \mathrm{SiCH}_{2} \mathrm{NHC}(\mathrm{S}) \mathrm{NHCH}_{3}(\mathrm{~B} 3 \mathrm{LYP} / \mathrm{6-31G}(d, p))$ дозволили встановити характеристичні смуги поглинання таутомерів. Так, наявність смуги поглинання при $1586 \mathrm{~cm}^{-1}$ (або при $1607 \mathrm{cм}^{-1}$ у разі фрагмента поверхні) вказує на присутність тіонної форми, а поява інтенсивної смуги поглинання при 1714 (1707) см ${ }^{-1}$ вказує на появу тіольної форми. Розрахунок величини повної енергї перехідного комплексу в вакуумі при $T=298 \mathrm{~K}$ і енергії активацї тіонтіольного таутомерного переходу для різних конформачій системи показав зниження активаційного бар'єру завдяки прищепленню тіосечовинних груп на поверхні кремнезему. Визначена конфігураиія перехідного комплексу.

Ключові слова: мезопоруватий кремнезем, тіон-тіольна таутомерія, ІЧ-спектроскопія, квантовохімічні розрахунки, метод функиіоналу електронної густини

\section{Тион-тиольная таутомерия тиомочевинных лигандов на поверхности кремнезема}

\author{
О.В. Смирнова, А.Г. Гребенюк, Г.И. Назарчук, Ю.Л. Зуб
}

Институт химии поверхности им. А.А. Чуйко Национальной академии наук Украинь ул. Генерала Наумова, 17, Киев, 03164, Украина, osmirnova@isc.gov.иа

Квантовохимические расчеты ИК-спектров тионной и тиольной форм N,N'-диметилтиомочевины и фрагмента поверхности кремнезема состава $(\mathrm{HO})_{3} \mathrm{SiCH}_{2} \mathrm{NHC}(\mathrm{S}) \mathrm{NHCH}_{3}(\mathrm{~B} 3 \mathrm{LYP} / 6-31 \mathrm{G}$ $(d, p))$ позволили установить характеристические полосы поглощения таутомеров. Так, наличие полосы поглощения при $1586 \mathrm{~cm}^{-1}$ (или при $1607 \mathrm{~cm}^{-1}$ в случае фрагмента поверхности) указывает на присутствие тионной формы, а появление интенсивной полосы поглощения при 1714 (1707) см $^{-1}$ указывает на появление тиольной формы. Расчет величины полной энергии переходного комплекса в 
вакууме при $T=298 \mathrm{~K}$ и энергии активации тион-тиольного таутомерного перехода для различных конформаций системы показал понижение активачионного барьера благодаря прививке тиомочевинных групп на поверхности кремнезема. Определена конфигурация переходного комплекса.

Ключевые слова: мезопористый кремнезем, тион-тиольная таутомерия, ИК-спектроскопия, квантовохимические расчеты, метод функиионала электронной плотности

\section{REFERENCES}

1. Pat. 7009 Japan, Int. Cl. 16B82. Susumu Kono et al. The mixed zinc salt and ethylene3-methylbutylene-bisdithiocarbamic acids method. (Publ. Feb. 28, 1972).

2. Salionov V.O., Buryak V.P., Panasenko O.I. et $a l$. Electronic absorption spectra and research thione-thiol tautomerism of 4-g-3-(thiophen-2yl)-1n-1,2,4-triazoles-5(4n)-thione, Actual Questions of Pharmaceutical and Medical Science and Practice, 3 (2012) 57 (in Ukrainian).

3. Erhitueva E.B., Zaharov V.I., Hramchihin A.V. (Eds.) Structure of thiotriazoles. St. Petersburg State Technological Institute (Technical University), 2014, 27 p. (in Russian).

4. Bershtein I.Ya., Kaminskij Yu.L. The Spectrometric Analysis in Organic Chemistry, Chemistry, Leningrad, 1986, 200 p. (in Russian).

5. Gazaliev A.M., Bakbardina O.V., Khrustalev $D . P$. Influence of solvent on the tautomeric equilibrium of cyclic thiourea. Proceedings of the University, Karaganda, 2010, 111 p. (in Russian).

6. Recent Advances in Density Functional Methods: abst., Ed. D. P. Chong., 1995, 413 p.

7. Delaere D. (Ed.) Thiol-thione tautomerism in thioformic acid: Importance of specific solvent interactions, J. Phys. Chem. A, 103 (1999) 171.

8. Tertykh V.A., Belyakova L.A. Chemical Reactions Involving Silica Surface, Kiev: Naukova Dumka, 1991, 264 p. (in Russian).

9. Nazarchuk G.I., Stolyarchuk N.V., Melnyk I.V. et al. The features of complexes formation on the silica surface containing thiourea group, Surface, 5 (2013) 102 (in Russian).

10. Nazarchuk G.I., Melnyk I.V., Zub Yu.L. et al. Mesoporous silica containing $\equiv \mathrm{Si}\left(\mathrm{CH}_{2}\right)_{3} \mathrm{NHC}(\mathrm{S}) \mathrm{NHC}_{2} \mathrm{H}_{5}$ functional groups in the surface layer, J. Colloid. Interf. Sci., 389 (2013) 115.

11. Nazarchuk G.I., Gona O.I., Zub Yu.L. Synthesis, structure and sorption properties of mesoporous silicas functionalized thiourea groups, Nanomaterials and Nanocomposites in Medicine, Biology, Ecology, Kiev, Naukova Dumka, 2011, 47 p. (in Russian).

12. Becke A.D. Density-functional thermochemistry. III. The role of exact exchange, J. Chem. Phys., 98 (1993) 5648.

13. Alex A. Granovsky, Firefly version 8, www http://classic.chem.msu.su/gran/firefly/index.html.

14. Schmidt M.W., Baldridge K.K., Boatz J.A. et al. General atomic and molecular electronic structure system, J. Comput. Chem., 14 (1993) 1347.

15. Miroshnichenko Yu.A., Beznosyk Yu.O., Smirnova O.V. et al. Quantum-chemical modeling of functionalized silica surface. Scientific notes of National Technical University of Ukraine «Kyiv Polytechnical Institute», 3 (2011) 141 (in Russian).

16. Miroshnichenko Yu.A., Beznosyk Yu.O., Smirnova O.V. et al. Quantum-chemical calculations of the fragments of the silica surface functionalized with nitrogen-, phosphor-, and sulfur-containing groups, The East European J. Adv. Tech., 14 (2012) 49 (in Russian).

Received 18.11.2014, accepted 23.04.2015 